\title{
Identificación de Serogrupos Patógenos de Leptospira en Canes Domésticos
}

\author{
Identification of Pathogenic Leptospira Serogroups in Domestic Dogs \\ Juan Siuce M. ${ }^{1}$, Sonia Calle E. ${ }^{1,2}$, Chris E. Pinto J. ${ }^{1}$, Gabriela Pacheco S. ${ }^{1}$, \\ Guillermo Salvatierra R. ${ }^{1}$
}

\section{Resumen}

La leptospirosis es una zoonosis bacteriana de gran impacto. Los perros y otros animales pueden infectarse, constituyendo un factor importante en la diseminación de la bacteria al humano. La infección es causada por cualquiera de los serovares patógenos de los 25 serogrupos de Leptospira spp. El estudio tuvo como objetivo identificar serogrupos de Leptospira spp presentes en perros con diagnóstico clínico presuntivo de leptospirosis de la ciudad de Lima. Se obtuvieron 305 muestras de suero sanguíneo de perros de 31 distritos de Lima Metropolitana. Se realizó la prueba de microaglutinación (MAT), estableciéndose títulos $\geq 1 / 100$ de serorreactividad como seropositivos. Se utilizaron cepas de referencia de los 25 serogrupos, incluyendo al nuevo serogrupo Iquitos, serovar varillal, cepa VAR10, aislado y reportado en casos humanos en Perú. Se detectaron 177 seropositivos $(58.0 \%), 31(10.2 \%)$ de los cuales fueron coaglutinaciones. Los serogrupos reaccionaron contra 18 serogrupos siendo los de mayor frecuencia: Iquitos (15.1\%), Tarassovi (12.1\%), Canicola (12.1\%), Australis (4.6\%), Icterohaemorrhagiae (4.3\%), Pomona (3.9\%), Mini (3.3\%) y Ballum (2.6\%). No se identificaron serorreactores en los serogrupos Bataviae, Celledoni, Hebdomadis, Lousiana, Panama, Ranarum y Sarmin.

Palabras clave: leptospirosis, Leptospira, MAT, serovar, serogrupo

\footnotetext{
${ }^{1}$ Laboratorio de Microbiología y Parasitología Veterinaria, Facultad de Medicina Veterinaria, Universidad Nacional Mayor de San Marcos, Lima, Perú

${ }^{2}$ E-mail: calleson@gmail.com
} 
Leptospirosis is an important bacterial zoonosis worldwide. Dogs and other animals can be infected, constituting an important role in the spread of the bacteria to humans. The infection is caused by any of the pathogenic serovars of the 25 serogroups of Leptospira spp. The aim of this study was to identify serogroups of Leptospira spp in dogs that had presumptive diagnosis of leptospirosis. A total of 305 serum samples were obtained from dogs in 31 districts of Lima. The microagglutination test (MAT) was performed, establishing $\geq 1 / 100$ seroreactivity titre as seropositive using reference strains of the 25 serogroups, including the new Iquitos serogroup, Varillal serovar, strain Var10, isolated and reported in human cases in Peru. The frequency of seropositive samples was $58.0 \%$ and $10.2 \%$ were co-agglutinations. The seropositive reacted against 18 serogroups and the most frequent were: Iquitos (15.1\%), Tarassovi (12.1\%), Canicola (12.1\%), Australis (4.6\%), Icterohaemorrhagiae (4.3\%), Pomona (3.9\%), Mini (3.3\%) and Ballum (2.6\%). There were no seroreactors to serogroups Bataviae, Celledoni, Hebdomadis, Lousiana, Panama, Ranarum, and Sarmin.

Key words: dog, leptospirosis, Leptospira, MAT, serovar, serogroup

\section{INTRODUCCIÓN}

La leptospirosis es una zoonosis de impacto global en la salud pública, con cerca de 500000 casos anuales y un rango de mortalidad de 5 a 20\% (Dupouey et al., 2013). La enfermedad se encuentra asociada a poblaciones con deficiencias sanitarias, especialmente en regiones tropicales (Adler y De la Pena Moctezuma, 2010; Evangelista y Cobum, 2010; Picardeu, 2013). Además, factores ambientales y naturales incrementan la presentación de la enfermedad en el verano o con el incremento de lluvias, así como con el fenómeno de El Niño e inundaciones (Levett, 2001; Pappas et al., 2008).

La enfermedad es causada por espiroquetas patógenas del género Leptospira, que produce en el humano fiebre alta, insuficiencia renal y hepática, que en casos graves conlleva la muerte dentro de una sintomatología conocida como el Síndrome de Weil (Adler y De la Pena Moctezuma, 2010). Los animales de producción, como el bovino, ovino, caprino, porcino y equino, padecen principalmente de problemas reproductivos y merma en su productividad (Ko et al., 2009). El perro presenta una alta sensibilidad a la enfermedad, con lesiones severas en riñones e hígado, y donde se han evidenciado cuatro formas de presentación o síndromes: ictérica, hemorrágica, urémica (enfermedad de Stuttgart) y reproductiva (Bolin, 1996), mientras que en gatos solo se han reportado casos leves o a través de infecciones experimentales (Hartmann et al., 2013).

El humano y los animales se infectan a través de las mucosas y superficies lesionadas o heridas y, en menor medida, por vía oral al exponerse directamente con orina de animales infectados o con fuentes de agua (acequias, riachuelos, lagunas, ríos). Los roedores son los principales diseminadores de la enfermedad, debido a su condición de portadores asintomáticos (Lau et al., 2010; Nakamura et al., 2013); sin embargo, los perros y otros animales pueden permanecer como portadores y eliminar continuamente la bacteria a través de la orina (Martins et al., 2012). 
Las leptospiras poseen un lento crecimiento en los medios de cultivo, requiriendo cerca de dos meses para el diagnóstico (Oliveira, 1999). Además, poseen más de 250 serovariedades patógenas, que por su similitud antigénica están agrupadas en 25 serogrupos. Protocolos actuales de PCR no definen el serovar/serogrupo infectante, a pesar de distinguir entre especies patógenas y saprófitas (Stoddard et al., 2009; Picardeau, 2013). Así, la prueba de Microaglutinación (MAT) es la herramienta diagnóstica de referencia internacional con mejor capacidad de distinguir y cuantificar anticuerpos contra determinado serogrupo (patógeno y saprófito). MAT emplea leptospiras vivas como antígeno, correspondientes a serovares de referencia, o mejor aún, serovares autóctonos o más frecuentes. A pesar de ello, MAT requiere determinar títulos de anticuerpos, signos clínicos del paciente y análisis bioquímicos para definir una posible exposición, infección reciente o enfermedad (Picardeau, 2013; OIE, 2014).

En el país se ha estudiado la leptospirosis en humanos y especies animales, con resultados positivos en todas las especies estudiadas (Céspedes et al., 2006). La leptospirosis canina es un hallazgo, o al menos un diagnóstico presuntivo, frecuente en las diversas clínicas veterinarias de Lima; de allí la importancia, tanto para el diagnóstico como para la producción de vacunas, de determinar los serovares/serogrupos circulantes de Leptospira spp en cada especie y zona geográfica. Además, la evaluación de la leptospirosis canina puede estimar el riesgo de la enfermedad para el humano, teniendo gran impacto en la salud pública (Martins et al., 2012). Por ello, el propósito del presente trabajo fue determinar los serogrupos de Leptospira spp presentes en perros de la zona de Lima con diagnóstico presuntivo de leptospirosis.

\section{Materiales y Métodos}

\section{Muestras}

Se obtuvieron muestras de sangre o suero de perros (Canis lupus familiares) con diagnóstico presuntivo de leptospirosis, remitidos por médicos veterinarios de clínicas veterinarias de Lima Metropolitana al Laboratorio de Bacteriología de la Facultad de Medicina Veterinaria, Universidad Nacional Mayor de San Marcos (UNMSM), Lima, Perú. En la historia clínica se describieron signos clínicos como fiebre, vómitos, hemorragias, oliguria, anuria, lumbalgia, dolor renal a la palpación, mialgias, diarrea, ictericia, convulsiones, poliuria, incremento de transaminasas y uremia.

El criterio de selección o inclusión de las muestras fue de animales mayores de seis meses de edad y sin vacunación contra leptospirosis o con más de seis meses desde la última vacunación. Se obtuvieron 305 muestras remitidas durante los años 2012 $(n=107), 2013(n=176)$ y $2014(n=22)$, procedentes de 31 distritos de la ciudad de Lima. Los distritos pertenecen a cuatro zonas geográficas: Lima Centro $(n=164)$, Lima Este $(n=89)$, Lima Norte $(n=20)$ y Lima Sur $(\mathrm{n}=32)$. Las muestras correspondieron a 193 machos y 112 hembras con edades de 0.5 a 3 años $(\mathrm{n}=87), 4$ a 7 años (n=91), 8 a 11 años $(n=78)$ y mayores de 11 años $(n=49)$.

\section{Muestras y Serología}

Las muestras de sangre fueron centrifugadas a $2500 \mathrm{rpm}$ durante 5 minutos. El suero resultante fue almacenado en microviales a $-20{ }^{\circ} \mathrm{C}$. La detección de anticuerpos contra leptospirosis se realizó con la prueba estándar de microaglutinación o aglutinación microscópica (MAT), según lo describe Faine (1982), y con las recomendaciones de la Organización Mundial de Sanidad Animal (OIE, 2014). 
Cuadro 1. Serovares de Leptospira spp utilizados en la prueba de microaglutinación (MAT)

\begin{tabular}{cllll}
\hline $\mathrm{N}^{\circ}$ & Especie & Serogrupo & Serovar & Cepa \\
\hline 1 & L. interrogans & Australis & Bratislava & Jez-bratislava \\
2 & L. interrogans & Autumnalis & Autumnalis & Akiyami A \\
3 & L. borgpetersenii & Ballum & Castellonis & Castellon 3 \\
4 & L. interrogans & Bataviae & Bataviae & Van Tienen \\
5 & L. interrogans & Canicola & Canicola & Hond Utrecht IV \\
6 & L. weilii & Celledoni & ND $^{1}$ & 2011/01963 \\
7 & L. kirschneri & Cynopteri & Cynopteri & 3522 c \\
8 & L. interrogans & Djasiman & Djasiman & Djasiman \\
9 & L. kirschneri & Grippotyphosa & Grippotyphosa & Moska V \\
10 & L. interrogans & Hebdomadis & Hebdomadis & Hebdomadis \\
11 & L. fainei & Hurstbridge & Hurstbridge & BUT6 \\
12 & L. interrogans & Icterohaemorrhagiae & Icterohaemorrhagiae & Verdun \\
13 & L. licerasiae & Iquitos & Varillal & VAR10 \\
14 & L. borgpetersenii & Javanica & Javanica & Poi \\
15 & L. noguchii & Louisiana & Louisiana & LUC1945 \\
16 & L. interrogans & Manhao & Lincang & L14 \\
17 & L. santarosai & Mini & Georgia & LT117 \\
18 & L. noguchii & Panama & Panama & CZ 214K \\
19 & L. interrogans & Pomona & Pomona & Pomona \\
20 & L. interrogans & Pyrogenes & pyrogenes & Salinem \\
21 & L. meyeri & Ranarum & Ranarum & ICF \\
22 & L. weilii & Sarmin & Sarmin & Sarmin \\
23 & L. interrogans & Sejroe & Hardjobovis & Sponselee \\
24 & L. santarosai & Shermani & Shermani & 1342 K \\
25 & L. borgpetersenii & Tarassovi & Tarassovi & Perepelitsin \\
\hline 1 & & & &
\end{tabular}

${ }^{1}$ No determinado

Se seleccionaron serovares de referencia internacional de cada uno de los 25 serogrupos patógenos establecidos (Cuadro 1). Los serovares fueron obtenidos de la Unidad de Espiroquetas del Instituto Pasteur, Francia. El criterio de selección fue tomado del manual de leptospirosis (OMS, 2008), incluyéndose en el presente estudio el nuevo serogrupo Iquitos, serovar Varillal cepa Var10 (Matthias et al., 2008).

Las leptospiras se cultivaron en tubos con $5 \mathrm{ml}$ del medio de cultivo líquido específico para leptospiras, el EllinghausenMcCullough-Johnson-Harris (EMJH), a $28^{\circ} \mathrm{C}$ durante 4-8 días. Se evaluó el crecimiento de cada serovar hasta obtener una cantidad de 1 a $2 \times 10^{8}$ leptospiras $/ \mathrm{ml}$, equivalente a una transmitancia de $60-70 \%$ utilizando un filtro de $400 \mathrm{~nm}$ (Espectofotómetro UNICO ${ }^{\mathrm{TM}}$, Modelo UV/VIS S2100). Luego se evaluó la viabilidad celular (movilidad) y la ausencia de contaminación, utilizando un microscopio de campo oscuro (OMS, 2008; OIE, 2014). Los tubos con un adecuado crecimiento se mantuvieron a temperatura ambiente hasta su uso en la prueba de MAT. Los serovares disponibles se utilizaron el mismo día de comprobarse su crecimiento óptimo. 


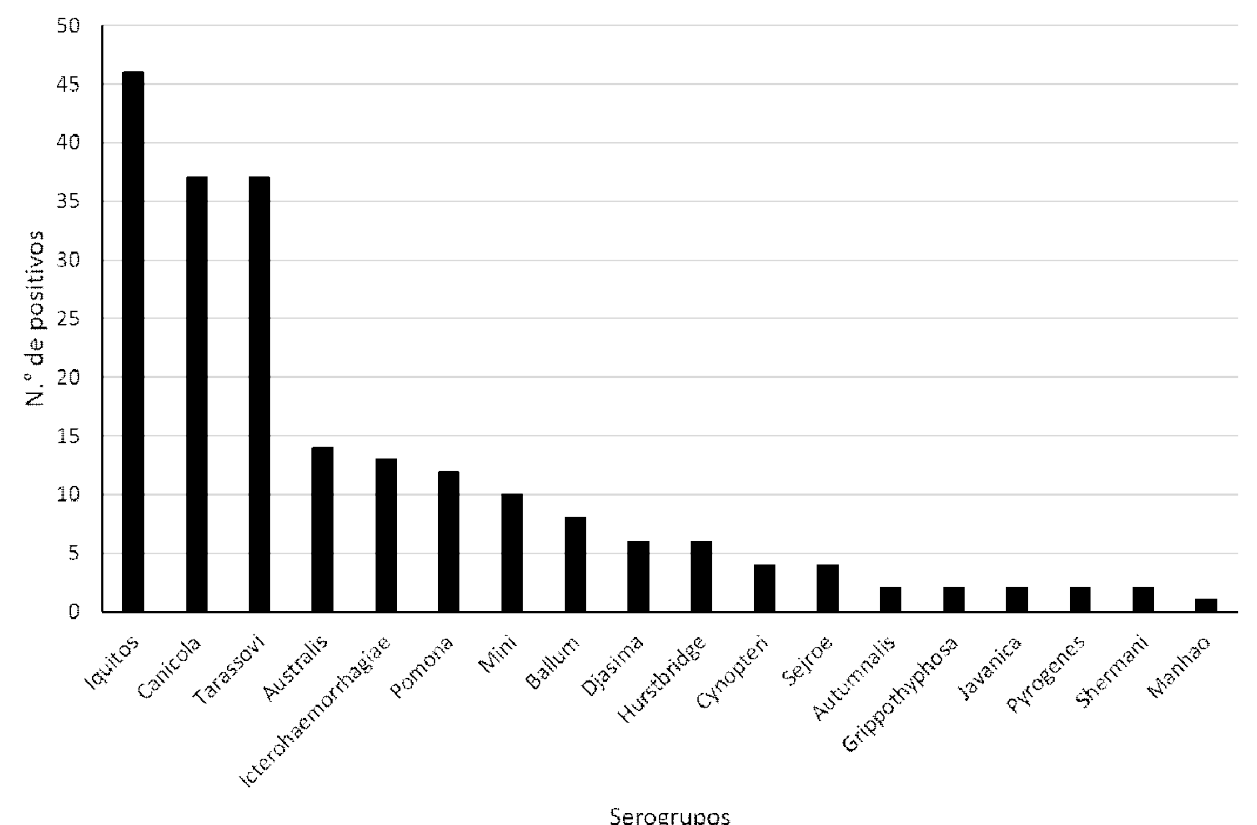

Figura 1. Seropositividad a Leptospira spp con la prueba de microaglutinación (MAT). Las barras muestra la frecuencia numérica de serogrupos patógenos de Leptospira spp (208 en 177 muestras positivas) en suero de perros con diagnóstico presuntivo de leptospirosis en Lima, Perú (2012-2014)

Las muestras se diluyeron en microplacas con solución salina fisiológica (ClNa $0.85 \%)$ para evaluar títulos de $1 / 100$, $1 / 200,1 / 400,1 / 800$ y $1 / 1600$ frente a antígenos vivos de leptospiras. Posterior a la incubación a $28{ }^{\circ} \mathrm{C}$ en oscuridad por $2 \mathrm{~h}$, los sueros con aglutinación no menor del $50 \%$ de las leptospiras (comparadas con el antígeno control) fueron considerados seropositivos o serorreactivos al respectivo título o dilución (INS, 2002; OIE, 2014).

Las muestras reactivas a un título igual o mayor de 1/100 a determinado serovar y de su respectivo serogrupo de Leptospira se consideraron como positivas (Faine et al., 1999; Picardeau, 2013). Las muestras reactivas a dos serogrupos con títulos iguales se consideraron como coaglutinaciones (OIE, 2014).

\section{Análisis Estadístico}

Los resultados fueron expresados en forma porcentual. Además, se estableció la relación entre los niveles de positividad con las variables procedencia, edad y sexo mediante la prueba de regresión logística utilizando el paquete estadístico STATA 11.

\section{Resultados}

El 58.0\% (177/305) de las muestras resultaron positivas a por lo menos uno de los 18 serogrupos (Cuadro 2, Fig. 1). No hubo respuesta contra los serogrupos Bataviae, Celledoni, Hebdomadis, Lousiana, Panama, Ranarum y Sarmin. 
Cuadro 2. Frecuencia de muestras positivas $^{1}$ a serogrupos de Leptospira spp $(\mathrm{n}=305)$

\begin{tabular}{lc}
\hline Serogrupo & $\begin{array}{c}\text { Frecuencia } \\
(\%)\end{array}$ \\
\hline Iquitos & 15.1 \\
Tarassovi & 12.1 \\
Canicola & 12.1 \\
Australis & 4.6 \\
Icterohaemorrhagiae & 4.3 \\
Pomona & 3.9 \\
Mini & 3.3 \\
Ballum & 2.6 \\
Djasiman & 2.0 \\
Hurtsbridge & 2.0 \\
Sejroe & 1.3 \\
Cynopteri & 1.3 \\
Autumnalis & 0.7 \\
Grippotyphosa & 0.7 \\
Pyrogenes & 0.7 \\
Javanica & 0.7 \\
Shermani & 0.7 \\
Manhao & 0.3 \\
\hline${ }^{1}$ 31 muestras fueron positivas a 2 serogrupos
\end{tabular}

Los títulos máximos de aglutinación de las muestras positivas a un serogrupo patógeno (146/177) de Leptospira spp fueron relativamente bajos en el caso de los serotipos más frecuentes; no obstante, algunos serovares alcanzaron títulos altos (Cuadro 3).

De los 177 seropositivos, 31 (10.2\%) fueron coaglutinaciones a serogrupos patógenos de Leptospira spp. De estos, los serogrupos que más participaron en las coaglutinaciones fueron Canicola, Iquitos e Icterohaemorrhagiae con títulos entre 1/100 y $1 / 800$ (Cuadro 4).

Los perros machos tuvieron mayor porcentaje de seropositividad (64.4\%) que las hembras. Asimismo, mayor seropositividad se presentó en perros con 4 a 11 años de edad (59.3\%) y en aquellos procedentes de Lima Centro $(52.0 \%)$. Los factores de riesgo se muestran en el Cuadro 5.

\section{Discusión}

El presente estudio es el primero en el país en evaluar los 25 serogrupos patógenos de Leptospira spp que han sido descritos. El $58.0 \%$ de perros seropositivos a Leptospira spp fue similar al 67\% (18/27) encontrado por Céspedes et al. (2003) en Madre de Dios, Perú, en caninos con diagnóstico presuntivo de leptospirosis. Asimismo, las frecuencias encontradas en clínicas veterinarias en Valdivia, Chile, de $14.8 \%$ en 400 perros evaluando ocho serotipos (Silva y Riedemann, 2007), de 18\% en Berlín, Alemania, en 329 perros sospechosos de Leptospirosis evaluando 12 serogrupos (Mayer-Scholl et al., 2013), y de $29.3 \%$ en Japón, en 283 perros con signos compatibles de leptospirosis (Koizumi $e t$ $a l ., 2013$ ) fueron seroprevalencias menores a las del presente trabajo, aunque en esos casos se emplearon menor número de serogrupos patógenos, disminuyendo la sensibilidad de los estudios.

Es importante considerar que los perros son susceptibles a la infección por la alta exposición al patógeno, inclusive en áreas urbanas, siendo el agua de regadío y de desagüe las principales fuentes de leptospiras debido a la eliminación continua de la bacteria por roedores y otros animales a través de la orina (Reis et al., 2008; Raghavan et al., 2011). Además, la ciudad de Lima cuenta con deficiencias en el sistema de alcantarillado y eliminación de desechos, principal factor asociado con leptospirosis humana en Perú y otros países (Oliveira et al., 2012), lo cual puede ser un factor que haya incrementado la frecuencia de perros positivos en el presente estudio (Sarkar et al., 2002; Krojgaard et al., 2009). 
Cuadro 3. Títulos de anticuerpos contra serogrupos patógenos de Leptospira spp mediante la prueba de microaglutinación (MAT) en muestras de suero positivos a un serogrupo $(\mathrm{n}=146)$ provenientes de canes con diagnóstico presuntivo de leptospirosis

\begin{tabular}{|c|c|c|c|c|c|c|}
\hline \multirow{2}{*}{ Serogrupo } & \multicolumn{5}{|c|}{ Títulos de anticuerpos } & \multirow{2}{*}{ Total } \\
\hline & $1 / 100$ & $1 / 200$ & $1 / 400$ & $1 / 800$ & $1 / 1600$ & \\
\hline Australis & 0 & 8 & 1 & 0 & 0 & 9 \\
\hline Autumnalis & 0 & 0 & 1 & 0 & 1 & 2 \\
\hline Ballum & 0 & 3 & 2 & 1 & 0 & 6 \\
\hline Canicola & 2 & 4 & 9 & 3 & 1 & 19 \\
\hline Cynopteri & 0 & 1 & 0 & 1 & 0 & 2 \\
\hline Djasiman & 1 & 2 & 0 & 2 & 0 & 5 \\
\hline Grippotyphosa & 0 & 1 & 0 & 1 & 0 & 2 \\
\hline Hurtsbridge & 0 & 2 & 2 & 1 & 0 & 5 \\
\hline Icterohaemorrhagiae & 3 & 1 & 2 & 0 & 0 & 6 \\
\hline Iquitos & 20 & 5 & 6 & 5 & 0 & 36 \\
\hline Javanica & 0 & 1 & 0 & 0 & 0 & 1 \\
\hline Mini & 0 & 3 & 1 & 2 & 0 & 6 \\
\hline Pomona & 2 & 1 & 4 & 0 & 0 & 7 \\
\hline Pyrogenes & 0 & 2 & 0 & 0 & 0 & 2 \\
\hline Manhao & 0 & 0 & 0 & 1 & 0 & 1 \\
\hline Sejroe & 1 & 2 & 1 & 0 & 0 & 4 \\
\hline Shermani & 0 & 0 & 1 & 0 & 0 & 1 \\
\hline Tarassovi & 2 & 8 & 18 & 2 & 2 & 32 \\
\hline Total & 31 & 44 & 48 & 19 & 4 & 146 \\
\hline
\end{tabular}

El creciente contacto con especies silvestres y el desplazamiento y comercialización de mascotas han resultado en cambios en la prevalencia de serogrupos de Leptospira spp, e inclusive en la emergencia de nuevos serogrupos, como se han documentado en Estados Unidos, Japón y países de Europa (Stokes et al., 2007; Ellis, 2010; Gautam et al., 2010; Koizumi et al., 2013).

Si bien se describe al perro como hospedero de mantenimiento del serovar Canicola, la mayoría de infecciones de perros en Estados Unidos en los últimos 20 años ha sido causada mayormente por los serovares Pomona, Bratislava y Grippotyphosa (Ward et al., 2004; Greene, 2006; Ghneim et al., 2007); mientras que en Valdivia, Chile, han sido Canicola, Icterohaemorrhagiae y Ballum (Silva y Riedemann, 2007), y en Japón y Europa han emergido o predominan serogrupos que en estudios previos tenían una mínima presencia e importancia epidemiológica (Gautam et al., 2010; Koizumi et al., 2013). Un estudio en Samoa Americana sugiere que existe una variación y emergencia de nuevos serogrupos cada 10 años (Lau et al., 2012), lo cual podría explicar las frecuencias del presente estudio y la presencia de serogrupos no reportados en estudios previos en perros y otras especies, tanto en Lima como a nivel nacional. 
Cuadro 4. Coaglutinaciones $(n=31)$ y sus respectivos títulos de anticuerpos mediante la prueba de microaglutinación (MAT) contra serogrupos patógenos de Leptospira spp en muestras de suero caninos con diagnóstico presuntivo de leptospirosis

\begin{tabular}{lcccccc}
\hline \multirow{2}{*}{ Serogrupos } & \multicolumn{5}{c}{ Títulos de anticuerpos } & \multirow{2}{*}{ Total } \\
\cline { 2 - 6 } Canicola-Australis & $1 / 100$ & $1 / 200$ & $1 / 400$ & $1 / 800$ & $1 / 1600$ & \\
Canicola-Ballum & 1 & 1 & 0 & 0 & 0 & 2 \\
Canicola-Cynopteri & 0 & 1 & 0 & 0 & 0 & 1 \\
Canicola-Hurstbridge & 0 & 0 & 1 & 0 & 0 & 1 \\
Canicola-Icterohaemorrhagiae & 0 & 0 & 1 & 0 & 0 & 1 \\
Canicola-Iquitos & 0 & 1 & 0 & 1 & 0 & 2 \\
Canicola-Mini & 1 & 3 & 0 & 0 & 0 & 4 \\
Canicola-Pomona & 3 & 0 & 0 & 0 & 0 & 3 \\
Canicola-Tarasovi & 1 & 2 & 0 & 0 & 0 & 3 \\
Icterohaemorrhagiae-Australis & 0 & 1 & 0 & 0 & 0 & 1 \\
Icterohaemorrhagiae-Iquitos & 0 & 1 & 1 & 0 & 0 & 2 \\
Icterohaemorrhagiae-Tarassovi & 2 & 0 & 0 & 0 & 0 & 2 \\
Iquitos-Australis & 0 & 0 & 1 & 0 & 0 & 1 \\
Iquitos-Pomona & 0 & 1 & 0 & 0 & 0 & 1 \\
Iquitos-Shermani & 0 & 1 & 0 & 0 & 0 & 1 \\
Iquitos-Tarassovi & 0 & 0 & 1 & 0 & 0 & 1 \\
Javanica-Tara ssovi & 0 & 0 & 1 & 0 & 0 & 1 \\
Mini-Djasiman & 1 & 0 & 0 & 0 & 0 & 1 \\
Pomona-Cynopteri & 0 & 0 & 0 & 1 & 0 & 1 \\
Tarassovi-Ballum & 0 & 0 & 1 & 0 & 0 & 1 \\
\hline Total & 0 & 0 & 0 & 1 & 0 & 1 \\
\hline
\end{tabular}

La mayor seropositividad correspondió al serogrupo Iquitos, serovar Varillal. Este serogrupo corresponde una especie nueva, denominada Leptospira liscerasiae, aislada de pacientes humanos de cuatro zonas geográficas distantes de la ciudad de Iquitos, en la amazonia peruana. Posterior a este hallazgo, se hizo una evaluación en pacientes febriles observándose en el $47 \%$ de las personas (Matthias et al., 2008). El empleo del nuevo serogrupo en el diagnóstico incrementó notablemente la detección de la enfermedad en varias localidades de la Amazonia (Céspedes et al., 2006). Incluso, el serovar Varillal fue encontrado en una persona del distrito de Puente Piedra, Lima con títulos de 1/200 (Platts-Mills et al., 2011), población que no presenta una relativa alta exposición a los factores de riesgo convencionalmente establecidos para contraer la leptospirosis; lo que estaría indicando que este serovar ya habría estado circulando en la ciudad de Lima. Esta ciudad tiene los factores de riesgo, tanto ambientales como biológicos y socio-económicos para la diseminación de la leptospirosis, y considerando el desplazamiento de mascotas y animales de producción y silvestres, se podría explicar la presencia y relativa alta fre- 
Cuadro 5. Factores de riesgo en caninos con diagnóstico presuntivo de leptospirosis en 305 muestras analizadas con la prueba de microaglutinación (MAT) contra serogrupos patógenos de Leptospira spp, medido a través del Odds Ratio

\begin{tabular}{|c|c|c|c|c|c|}
\hline \multirow{2}{*}{ Variable } & & \multicolumn{2}{|c|}{ Seropositivos } & \multirow{2}{*}{$\begin{array}{c}\mathrm{OR}^{1 \mathrm{a}} \\
\text { ajustado }\end{array}$} & \multirow[t]{2}{*}{ IC $95 \%^{2}$} \\
\hline & & Positivos & $\%^{\mathrm{a}}$ & & \\
\hline \multirow[t]{2}{*}{ Sexo } & Macho & 114 & 64.4 & 1 & \\
\hline & Hembra & 63 & 35.6 & 0.75 & $0.5-1.2$ \\
\hline \multirow[t]{4}{*}{ Edad } & $<3$ años & 46 & 26.0 & 1 & \\
\hline & 4-7 años & 53 & 29.9 & 1.39 & $0.7-2.2$ \\
\hline & 8-11 años & 52 & 29.4 & 1.96 & $0.9-3.4$ \\
\hline & $>11$ años & 26 & 14.7 & 0.98 & $0.5-2.0$ \\
\hline \multirow[t]{4}{*}{ Procedencia } & Lima Norte & 7 & 4.0 & 1 & \\
\hline & Lima Centro & 92 & 52.0 & 0.24 & $0.9-6.2$ \\
\hline & Lima Sur & 21 & 11.9 & 3.54 & $1.1-11.5^{3}$ \\
\hline & Lima Este & 57 & 32.2 & 3.30 & $1.2-9.1^{3}$ \\
\hline
\end{tabular}

${ }^{a}$ Odds ratio ajustado (análisis multivariado - regresión logística)

${ }^{2}$ Intervalo de confianza

${ }^{3}$ Factor de riesgo en análisis multivariado $(p<0.05)$

cuencia en la población canina del presente estudio.

Los serogrupos Canicola, Iquitos e Icterohaemorrhagiae fueron los más frecuentes en las coaglutaciones entre ellos y con otros serogrupos $(10.2 \%)$, cuyo porcentaje alto se explicaría por el uso de una amplia variedad de serogrupos patógenos, incrementando la sensibilidad de la prueba. Los títulos de las coaglutinaciones variaron desde $1 / 100$ hasta $1 / 800$. Las coaglutinaciones suelen deberse a infecciones por dos o más serovares patógenos; sin embargo, en algunos casos los perros pudieron pasar por una infección subaguda o crónica a determinado serovar y luego se infectaron con otro serovar de distinto serogrupo. Asimismo, también puede deberse a reacciones cruzadas (Ferreira Neto et al., 1997; Andre-Fontaine, 2006). Coaglutinaciones han sido demostradas entre el serogrupo Iquitos con otro serogrupo patógeno (18\%, Matthias et al., 2008). Igualmente, en Chile se obtuvo $4.3 \%$ (Silva y
Riedemann, 2007) y en Colombia se determinó $2.0 \%$ de coaglutinaciones (Silva-Molano et al., 2008).

La determinación de la edad y el sexo como factores de riesgo para la infección con leptospiras ha sido observado en Lima por Huerta et al. (2013), quienes, además, consideran que el tamaño del perro es otro factor de riesgo en caninos. Esto se explicaría por la mayor exposición de los perros adultos, machos y de mayor tamaño que tienen mayor acceso a la calle que perros jóvenes, hembras (para evitar la preñez) y pequeños.

\section{ConClusiones}

- Las infecciones de Leptospira spp en perros de la ciudad de Lima son causadas por 18 de los 25 serogrupos patógenos: Australis, Autumnalis, Ballum, Canicola, Cynopteri, Djasiman, Grippotyphosa, Hurstbridge, Icterohaemorrha- 
giae, Iquitos, Javanica, Manhao, Mini, Pomona, Pyrogenes, Sejroe, Shermani y Tarassovi.

- La procedencia del perro fue un factor de riesgo en la presentación de leptospirosis en perros con diagnóstico presuntivo de leptospirosis.

\section{Agradecimientos}

Los autores agradecen el financiamiento del estudio al Consejo Superior de Investigaciones de la UNMSM y el apoyo del técnico Jorge Bautista en la realización del presente estudio.

\section{Literatura Citada}

1. Adler B, De la Peña Moctezuma A. 2010. Leptospira and leptospirosis. Vet Microbiol 140: 287-296. doi: 10.1016/ j.vetmic.2009.03.012

2. André-Fontaine G. 2006. Canine leptospirosis - Do we have a problem? Vet Microbiol 117: 19-24. doi: 10.1016/ j.vetmic.2006.04.005

3. Bolin C. 1996. Diagnosis of leptospirosis: a reemerging disease of companion animals. Semin Vet Med Surg (Small Anim) 11: 166-171.

4. Céspedes M, Ormaeche M, Condori P, Balda L, Glenny M. 2003. Prevalencia de leptospirosis y factores de riesgo en personas con antecedentes de fiebre en la provincia de Manu, Madre de Dios, Perú. Rev Peru Med Exp Salud Pública 20: 180-185.

5. Céspedes M, Balda L, González D, Tapia R. 2006. Situación de la leptospirosis en el Perú: 1994-2004. Rev Peru Med Exp Salud Pública 23: 52-62.

6. Dupouey J, Faucher B, Edouard S, Richet H, Kodjo A, Drancourt M, Davoust B. 2013. Human leptospirosis: an emerging risk in Europe? Comp Immunol Microbiol Infect Dis 37: 77-83. doi: 10.1016/ j.cimid.2013.12.002.
7. Ellis WA. 2010. Control of canine leptospirosis in Europe: time for a change? Vet Rec 167: 602-605. doi: 10.1136/vr.c4965

8. Evangelista KV, Coburn J. 2010. Leptospira as an emerging pathogen: a review of its biology, pathogenesis and host immune responses. Future Microbiol 5: 1413-1425. doi: 10.2217/fmb.10.102

9. Faine S, Adler B, Bolin C, Perolat P. 1999. Leptospira and leptospirosis. En: Faine $S$ (ed). Methods. $2^{\text {nd }}$ ed. Melbourne, Australia: MedScience. $p$ 169-184.

10. Faine S. 1982. Guidelines for the control of leptospirosis. WHO Offset Publication N. ${ }^{\circ}$ 67. Geneva: WHO. 98 p.

11. Ferreira Neto JS, Vasconcellos SA, Ito $F H$, Moretti AS, Camargo CA, Sakamoto SM, et al. 1997. Leptospira interrogans serovar icterohaemorrhagiae seropositivity and the reproductive performance of sows. Prev Vet Med 31: 87-93. doi: 10.1016/S0167-5877(96)011208

12. Gautam R, Guptill LF, Wu CC, Potter A, Moore GE. 2010. Spatial and temporal-spatial clustering of overall and serovar-specific Leptospira microscopic agglutination test (MAT) seropositivity among dogs in the United States from 2000 through 2007. Prev Vet Med 96: 122-131. doi: 10.1016/j.prevetmed. 2010.05.017

13. Ghneim GS, Viers JH, Chomel BB, Kass PH, Descollonges DA, Johnson ML. 2007. Use of a case-control study and geographic information systems to determine environmental and demographic risk factors for canine leptospirosis. Vet Res 38: 37-50.

14. Greene CE. 2006. Laboratory diagnosis of canine leptospirosis and babesiosis. En: 24 $4^{\text {th }}$ Annual ACVIM Forum. Louisville, KY, USA.

15. Hartmann K, Egberink H, Pennisi $M G$, Lloret A, Addie D, Belák S, Boucraut-Baralon C, et al. 2013. Leptospira species infection in cats: 
ABCD guidelines on prevention and management. J Feline Med Surg 15: 576581. doi: 10.1177/1098612X13489217

16. Huerta C. Chilón V, Díaz D. 2013. Estudio de caso-control para evaluar factores de riesgo en la presentación de leptospirosis canina en la ciudad de Lima. Rev Inv Vet Perú 24: 111-117. doi: 10.15381/rivep.v24i1.1674

17. [INS] Instituto Nacional de Salud del Perú. 2002. Manual de procedimientos bacteriológico y serológico para el diagnóstico de la leptospirosis. Lima: Ministerio de Salud, Instituto Nacional de Salud. $53 \mathrm{p}$.

18. Ko AI, Goarant C, Picardeau M. 2009. Leptospira: the dawn of the molecular genetics era for an emerging zoonotic pathogen. Nat Rev Microbiol 7: 736-747. doi: 10.1038/nrmicro2208

19. Koizumi N, Muto M, Akachi S, Okano S, Yamamoto S, Horikawa K, Harada $S$, et al. 2013. Molecular and serological investigation of Leptospira and leptospirosis in dogs in Japan. J Med Microbiol 62: 630-636. doi: 10.1099/ jmm.0.050039-0

20. Krojgaard LH, Villumsen $S$, Markussen MDK, Jensen JS, Leirs H, Heiberg AC. 2009. High prevalence of Leptospira spp. in sewer rats (Rattus norvegicus). Epidemiol Infect 137: 1586-1592. doi: 10.1017/S0950268809002647

21. Lau C, Smythe L, Weinstein P. 2010. Leptospirosis - an emerging disease in travellers. Travel Med Infect Dis 8: 33 39. doi: 10.1016/j.tmaid.2009.12.002

22. Levett PN. 2001. Leptospirosis. Clin Microbiol Rev 4: 296-326.

23. Martins G, Penna B, Lilenbaum W. 2012. The dog in the transmission of human leptospirosis under tropical conditions: victim or villain? Epidemiol Infect 140: 207-208. doi: 10.1017/ S0950268811000276
24. Matthias MA, Ricaldi JN, Cespedes M, Diaz MM, Galloway RL, Saito M, Steigerwalt $A G$, et al. 2008. Human leptospirosis caused by a new, antigenically unique Leptospira associated with a Rattus species reservoir in the Peruvian Amazon. PLoS Negl Trop Dis 2: e213. doi: 10.1371/journal.pntd.0000213

25. Mayer-Scholl A, Luge E, Draeger A, Nöckler K, Kohn B. 2013. Distribution of Leptospira serogroups in dogs from Berlin, Germany. Vector Borne Zoonotic Dis 13: 200-202. doi: 10.1089/ vbz.2012.1121

26. Nakamura I, Hang'ombe BM, Sawa H, Kobayashi S, Orba Y, Ishii A, Thomas Y, et al. 2013. Cross-reactivity of secondary antibodies against African rodents and application for serosurveillance. J Vet Med Sci 75: 819-825. doi: $10.1292 / j v m s .12-0471$

27. [OIE] Organización Mundial de Sanidad Animal. 2014. Manual de las pruebas de diagnóstico y de las vacunas para los animales terrestres. Cap. 2.1.9 Leptospirosis. Paris: OIE. [Internet]. Disponible en: http://www.oie.int/es/normas-internacionales/manual-terrestre/ acceso-en-linea/

28. Oliveira SJ. 1999. Nova ameaça à reprodução em suínos, além da leptospirose? Hora Vet 19: 87-90.

29. Oliveira Lavinsky M, Said RA, Strenzel GM, Langoni H. 2012. Seroprevalence of anti-Leptospira spp antibodies in dogs in Bahia, Brazil. Prev Vet Med 106: 79-84. doi: 10.1016/ j.prevetmed.2012.03.015

30. [OMS] Organización Mundial de la Salud. 2008. Leptospirosis humana: guía para el diagnóstico, vigilancia y control. Rio de Janeiro: OMS-OPS. 127 p.

31. Pappas G, Papadimitriou P, Siozopoulou V, Christou L, Aktritidis $N$. 2008. The globalization of leptospirosis: worldwide incidence trends. Int J Infect Dis 12: 351-357. 
32. Picardeau M. 2013. Diagnosis and epidemiology of leptospirosis. Med Mal Infect 43: 1-9. doi: 10.1016/j.medmal. 2012.11.005

33. Platts-Mills J, LaRochelle P, Campos K, Vinetz J, Gotuzzo E, Ricaldi J. 2011. Seroprevalencia de leptospirosis en Puente Piedra, Lima en el año 2006. Rev Peru Med Exp Salud Pública 28: 273-276.

34. Raghavan $R$, Brenner K, Higgins $J$, Van der Merwe D, Harkin KR. 2011. Evaluations of land cover risk factors for canine leptospirosis: 94 cases (20022009). Prev Vet Med 101: 241-249. doi: 10.1016/j.prevetmed.2011.05.010

35. Reis RB, Ribeiro GS, Felzemburgh RDM, Santana FS, Mohr S, Melendez AXTO, Queiroz A, et al. 2008. Impact of environment and social gradient on Leptospira infection in urban slums. PLoS Neglect Trop Dis 2:e228. doi: 10.1371/journal.pntd.0000228

36. Sarkar U, Nascimento SF, Barbosa R, Martins R, Nuevo H, Kalafanos I, Grunstein I, et al. 2002. Populationbased case-control investigation of risk factors for leptospirosis during an urban epidemic. Am J Trop Med Hyg 66: 605610.

37. Silva R, Riedemann F. 2007. Seroprevalencia de leptospirosis canina en perros atendidos en clínicas veterinarias, mediante aglutinación microscópica y comparación con las técnicas de aislamiento e inmunofluorescencia indirecta. Arch Med Vet 39: 269-274. doi: 10.4067/S0301-732X2007000300011

38. Silva-Molano R, Castro F, Montoya J, Loaiza-Echeverri A. 2008. Estudio de seroprevalencia de leptospirosis canina en Manizales - Colombia, mediante aglutinación microscópica (MAT). Vet Zootec 2(2): 35-39.

39. Stoddard RA, Gee JE, Wilkins PP, McCaustland K, Hoffmaster AR. 2009. Detection of pathogenic Leptospira spp through TaqMan polymerase chain reaction targeting the LipL32 gene. Diagn Microbiol Infect Dis 64: 247-255. doi: 10.1016/j.diagmicrobio.2009.03.014

40. Stokes JE, Kaneene JB, Schall WD, Kruger JM, Miller R, Kaiser L. Bolin CA. 2007. Prevalence of serum antibodies against six Leptospira serovars in healthy dogs. J Am Vet Med Assoc 230: 1657-1664. doi: 10.2460/ javma.230.11.1657

41. Ward MP, Guptill LF, Wu CC. 2004. Evaluation of environmental risk factors for leptospirosis in dogs: 36 cases (19972002). J Am Vet Med Assoc 225: 72-77. doi: 10.2460/javma.2004.225.72 